\title{
PRÉSENTATION D'UN NOUVEL ABAQUE POUR DÉTERMINER LES LIMITES DE CONFIANCE DU PEUPLEMENT LE PLUS PROBABLE
} ESTIMÉ PAR LA MÉTHODE DE PETERSEN

\author{
P. Lamarque \\ Centre de Recherches Hydrobiologiques \\ I.N.R.A., B.P. 3 Saint Pée sur Nivelle - 64310 ASCAIN
}

RESUME

L'abaque présenté est facile et rapide d'emploi. II fournit des limites de confiance symétriques du peuplement le plus probable ainsi que le niveau de précision de l'inventaire, ce qui permet sur le terrain de décider de la prolongation éventuelle des pêches.

\section{ABSTRACT}

The new abacus is quick and easy to utilize, it provides symmetrical confidence limits of the most probable fish population, as well as the precision level of the inventory, which enables to decide, on the field. the possible extension of the fishing operations.

Article available at http://www.kmae-journal.org or http://dx.doi.org/10.1051/kmae:1978011 


\section{1. - INTRODUCTION}

Lorsqu'on estime un peuplement de poissons par la méthode de PETERSEN, il est nécessaire, pour calculer ce peuplement et ses limites de confiance, de le décomposer en peuplements fragmentaires par espèces et par catégories de longueur, l'efficacité de la pêche variant avec ces facteurs (CUINAT et al., 1975 a et b).

II en résulte un nombre considérable de calculs nécessitant un temps important, même si on utilise un programme d'ordinateurs ou des calculateurs de bureau. C'est pour cette raison que l'on préfère utiliser des abaques plutôt que de faire des calculs d'écart-types.

Les abaques actuellement en usage présentant certains inconvénients, nous avons cherchè à les améliorer et à les simplifier.

\section{2. - METHODES ACTUELLEMENT UTILISEES POUR LA DETERMINATION DES LIMITES DE CON:IANCE AVEC UNE SECURITE DE 95 POUR CENT}

\section{1. - Ecart-type d'une proportion (TIMMERMANS, 1957)}

Nous adopterons la symbolique suivante :

$P$ : peuplement le plus probable

$P^{\prime}$ : peuplement le plus probable assorti de la limite supérieure

$\mathrm{P}^{\prime \prime}$ : peuplement le plus probable assorti de la limite inférieure

$\mathrm{m}$ : nombre de poissons marqués et remis à l'eau après la première pêche

$u$ : nombre de poissons non marqués capturés à la seconde pêche

$r$ : nombre de poissons marqués capturés à la seconde pêche

$n$ : nombre total de poissons capturés à la seconde pêche $(n=u+r)$

$\mathrm{e}$ : efficacité de la première pêche.

En prenant en compte l'écart-type de la proportion e, on obtient :

$$
\begin{array}{r}
P^{\prime}=\frac{m}{e-2 \sqrt{e}} \quad \text { et } P^{\prime \prime}=\frac{m}{e+2 \sqrt{e}} \\
\text { où : } \quad \sqrt{e}=\sqrt{\frac{e(1-e)(P-n)}{n(P-1)}}
\end{array}
$$

On conçoit que cette méthode est particulièrement longue à utiliser quand le peuplement n'est pas homogène et qu'il est nécessaire de le subdiviser.

2.2. - Abaques de CLOPPER et PEARSON (1934, d'après CUINAT 1975, a et b) et de CUINAT (1975, a et b)

Les abaques de CLOPPER et PEARSON (fig. 1) ont été établis pour des peuplements importants. Ils fournissent pour les peuplements relativement faibles des limites de confiance trop écartées.

C'est pourquoi CUINAT (fig. 2) les a modifiés pour les petits cours d'eau à truites, lorsque l'effectif de la première pêche est sensiblement égal à celui de la seconde $(m \simeq n)$.

Cependant ces abaques présentent trois inconvénients:

- ils sont peu précis lorsque le nombre de poissons est supérieur à 200 , en raison de leur configuration graphique; 


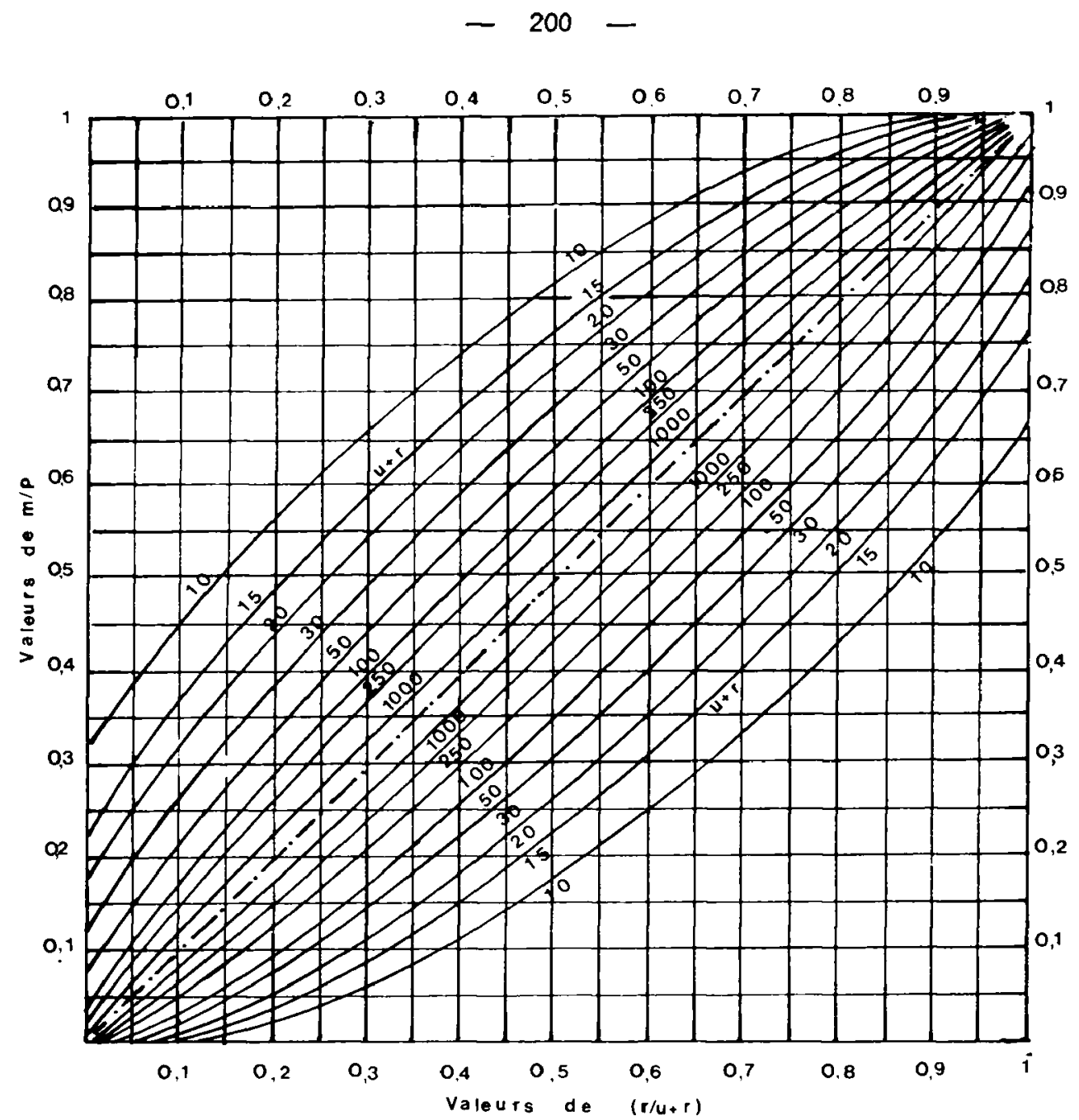

Figure 1 - ABAQUE de CLOPPER et PEARSON (1934, d'après CUINAT, 1975 a et b) Signification des symboles :

$m=$ nombre de poissons, d'une espèce donnée et d'une catégorie de longueur, marqués et remis à l'eau après la première pêche

$r=$ nombre de poissons des mêmes espèce et catégorie de longueur. marqués, capturés au cours de la seconde pêche

$u=$ nombre de poissons des mêmes espèce et catégorie de longueur. non marqués et capturés au cours de la seconde pèche

$\mathrm{P}=$ nombre le plus probable de ces poissons évalué par la méthode de PETERSEN : $P=\frac{m}{r /(u+r)}=\frac{m(u+r)}{r}$

Utilisation de l'abaque. Exemple :

Soit $m=120, r=60$ et $u=40 . r / u+r=0,6$ et $P$ (le plus probable) $=\mathrm{m} / 0,6=200$. Les intersections des deux courbes $(u+r)=100$ avec la ligne verticale tirée du point 0.6 sur l'axe des $X$, ont respectivement 0,49 et 0,69 pour ordonnées. Les limites de confiance autour de $P=200$ sont donc: $P^{\prime}=120 / 0,49=245$ et $P^{\prime \prime}=120 / 0,69=174$. 


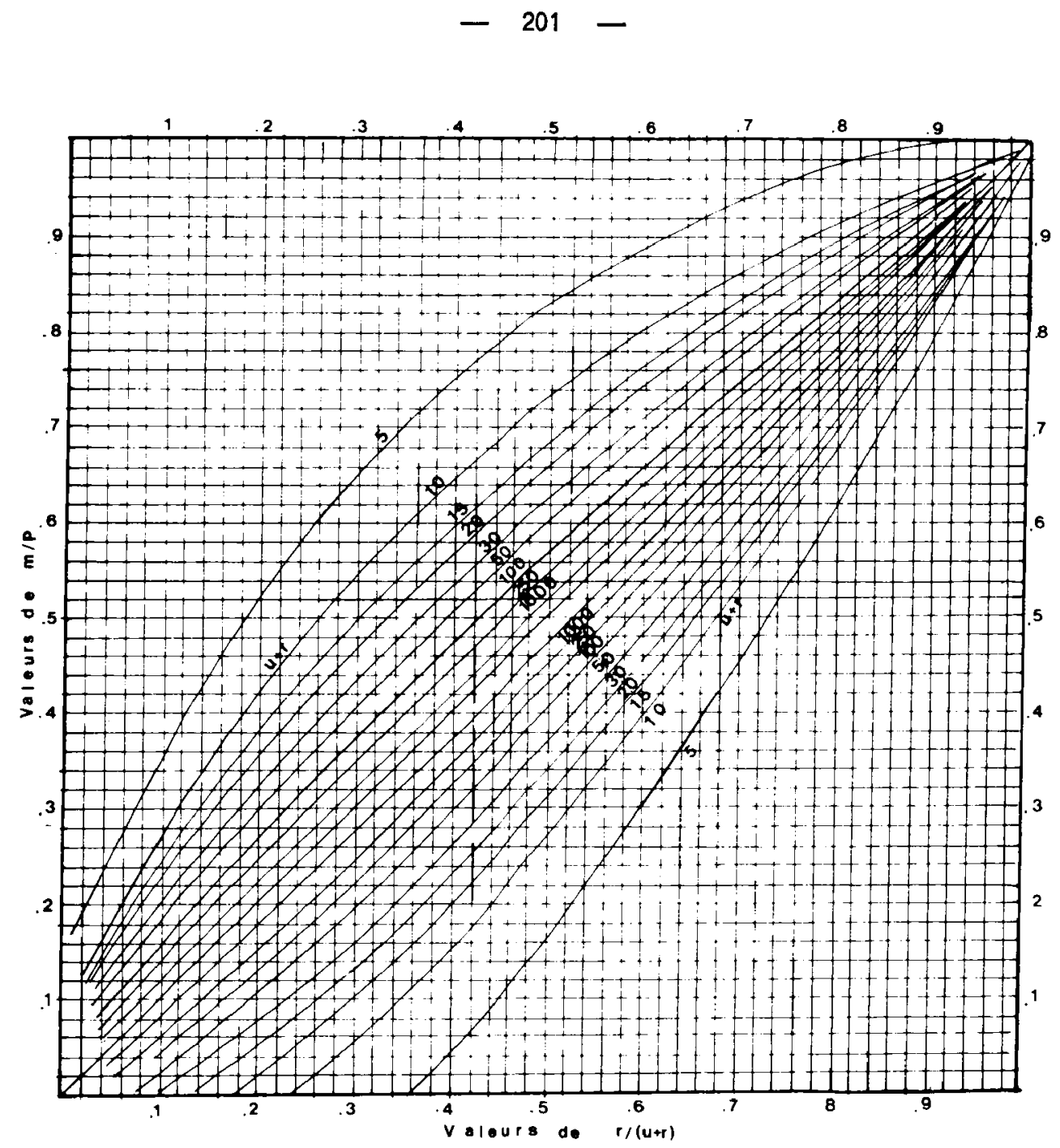

Figure 2 - ABAQUE DE CUINAT (1975, a et b)

Mêmes symboles et même mode d'utilisation que dans ia figure 1.

- ils ne fournissent pas de limites de confiance symétriques du peuplement le plus probable (de méme que le calcul de l'écart-type):

— ils sont plus difficiles à lire que l'abaque qui va être proposé.

\section{3. - ETABLISSEMENT DE L'ABAQUE DE LAMARQUE}

Nous conserverons la symbolique ci-dessus mentionnée. CHAPMAN (1951), pour réduire le biais des petits échantillons, a introduit un correctif dans la formule de PETERSEN, qui ne modifie d'ailleurs les résutats que de très peu.

Au lieu de la formule: $P=\frac{m n}{r}$, 
i) a proposé: $P=\frac{(m+1)(n+1)}{(r+1)}$.

A partir de cette relation, on peut calculer la variance du peuplement en utilisant la formule suivante (VIN!CENT, 1971):

$$
\text { Var. } P=\frac{P^{2} u}{(n+1)(r+2)}
$$

soit pour 2 écart-types:

$$
2 \backslash \overline{\frac{P^{2} u}{(n+1)(r+2)} \text {. }} \text {. }
$$

Les peuplements assortis de leurs limites de confiance sont:

$$
\begin{aligned}
& P^{\prime}=P+2 \sqrt{\frac{P^{\prime 2} u}{(n+1)(r+2)}} \\
& \text { et } P^{\prime \prime}=P-2 \sqrt{\frac{P^{2} u}{(n+1)(r+2)}}
\end{aligned}
$$

On obtient ainsi des limites symétriques du peuplement estimé.

$S$, maintenant, on considere les deux écarts-types comme une proportion $p$ du peupiement estimé (précision de la mesure), on peut écrire :

$$
2 \backslash \frac{P^{2}(n-r)}{(n+1)(r+2)}=p P \quad(u \text { est égal à } n-r) \text {. }
$$

En développant et en simplifiant, il vient:

$$
\frac{4(n-r)}{(n+1)(r+2)}=p^{2} \text {, relation independante de } P \text {. }
$$

On introduit dans cette relation le rapport $\stackrel{r}{-}$ (efficacite de la première pêche) et l'on obtient une fonction de la forme:

$$
n=f\left(\frac{r}{n}\right)
$$

Pour construire labaque, on programme les différentes valeurs de $p$, et pour chaque valeur de $p$ les differentes valeurs de $n$.

On obtient ainsi les valeurs de $r$ qui permettent de calculer le rapport -

II suffit ensuite de tracer les diverses courbes $p$ en portant en abscisse les $r$

valeurs - et en ordonnée les valeurs $n$ correspondantes (fig. 3). 


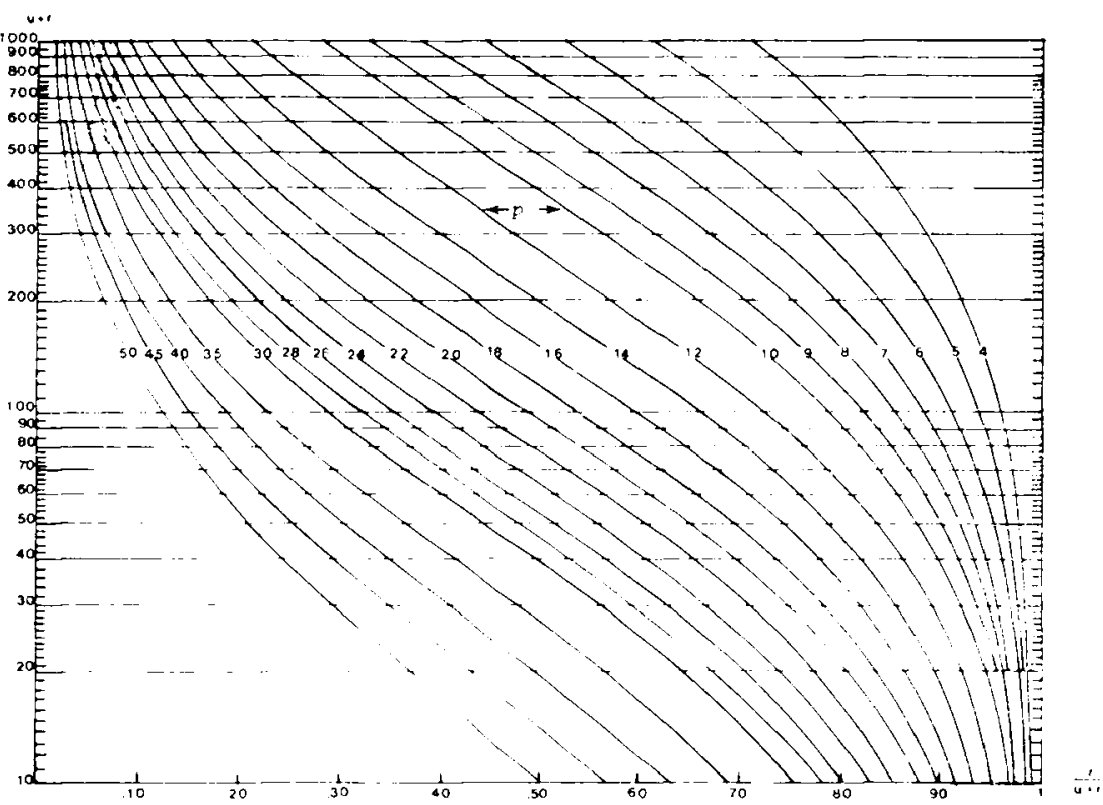

\section{Figure 3 - ABAQUE DE LAMARQUE}

$m$ = nombre de poissons d'une espèce donnée et d'une catégorie de longueur, marquès et remis à l'eau après la première pêche

$r=$ nombre de poissons d'une espèce donnée et d'une catégorie de longueur, marqués et capturés au cours de la seconde pêche

$u=$ nombre de poissons d'une espèce donnée et d'une catégorie de longueur, non marqués et capturés au cours de la seconde pêche

$P=$ nombre le plus probable de ces poissons évalué par la méthode de PETERSEN modifiée par CHAPMAN

$$
\begin{aligned}
& P=\frac{(m+1)(u+r+1)}{(r+1)} \\
& P=\text { précision de la mesure en pour cent. }
\end{aligned}
$$

Utilisation de l'abaque. Exemple:

Chercher le point d'ordonnée $(u+r)$ et d'abscisse $\frac{r}{(u+r)}$. La courbe de

précision $p$ qui contient ce point donne les limites de confiance en pourcentage du peuplement avec une sécurité de 95 pour cent.

Soit : $P=200 ; u+r=100: \frac{r}{(u+r)}=0,6$. La courbe correspondante

est 16 , ce qui signifie que les limites de confiance sont respectivement $\pm 0,16 \mathrm{P}: \mathrm{P}^{\prime}=\mathrm{P}+0,16 \mathrm{P}=232$ et $\mathrm{P}^{\prime \prime}=\mathrm{P}-0,16 \mathrm{P}=168$. 


\section{4. - COMPARAISON DES LIMITES DE CONFIANCE OBTENUES PAR LES DIVER- SES METHODES}

Les limites de confiance fournies par le nouvel abaque sont toujours plus resserrées que celles fournies par l'abaque de CLOPPER et PEARSON, mais un peu plus étendues que par les autres méthodes. Cependant pour les petits peuplements, elles sont très proches de celles données par l'écart-type de la proportion et de celles de l'abaque de CUINAT, ainsi que le montrent les exemples du tableau 1.

Tableau 1 - Exemples de peuplements assortis de leurs limites de confiance établies par les diverses méthodes.

\begin{tabular}{|c|c|c|}
\hline & Petits peuplements & Grands peuplements \\
\hline $\begin{array}{l}\text { Résultats de } \\
\text { l'inventaire }\end{array}$ & $\begin{array}{l}P=20 ; m=12 ; r=6 \\
u=4 ;(u+r)=10 \\
r /(u+r)=0,6\end{array}$ & $\begin{array}{l}P=400 ; m=240 ; r=120 \\
u=80 ;(u+r)=200 \\
r /(u+r)=0,6\end{array}$ \\
\hline Abaque de CUINAT & $\mid \begin{array}{l}P^{\prime}=30 \\
P^{\prime \prime}=15\end{array}$ & $\begin{array}{l}P^{\prime}=436 \\
P^{\prime \prime}=369\end{array}$ \\
\hline $\begin{array}{l}\text { Ecart-type d'une } \\
\text { proportion }\end{array}$ & $\begin{array}{l}P^{\prime}=32 \\
P^{\prime \prime}=14\end{array}$ & $\begin{array}{l}P^{\prime}=435 \\
P^{\prime \prime}=369\end{array}$ \\
\hline $\begin{array}{l}\text { Abaque de CLOPPER } \\
\text { et PEARSON }\end{array}$ & $\begin{array}{l}P^{\prime}=48 \\
P^{\prime \prime}=13\end{array}$ & $\begin{array}{l}P^{\prime}=470 \\
P^{\prime \prime}=363\end{array}$ \\
\hline $\begin{array}{l}\text { Nouvel abaque } \\
\text { de LAMARQUE }\end{array}$ & $\mid \begin{array}{l}P^{\prime}=29 \\
P^{\prime \prime}=11 \\
\text { précision : } 0,42\end{array}$ & $\left\{\begin{array}{l}\mathrm{P}^{\prime}=446 \\
\mathrm{P}^{\prime \prime}=354 \\
\text { précision : } 0,11\end{array}\right.$ \\
\hline
\end{tabular}

Observation: A l'exception de l'abaque de CLOPPER et PEARSON pour les petits peuplements, les différences enregistrées entre les diverses méthodes sont négligeables en regard de l'imprécision de la méthode d'inventaire elle-même.

\section{5. - Lecture de l'abaque de lamarQue}

On place une règle graduée au point d'ordonnée $n=u+r$.

On lit sur la règle l'abscisse $\frac{r}{u+r}$.

La courbe de précision $p$ qui contient ce point fournit les limites de confiance.

Exemple:

$P=400 ; n=u+r=200 ; \frac{r}{u+r}=0,6$.

La courbe de précision qui contient le point d'ordonnée 200 et d'abscisse 0,6 est : 11,5 . 
Les peuplements supérieur et inférieur sont avec une sécurité de 95 pour cent :

$$
\begin{aligned}
& P^{\prime}=400+(0,115 \times 400)=446 \\
& P^{\prime \prime}=400-(0,115 \times 400)=354
\end{aligned}
$$

\section{6. - INTERET DE CE NOUVEL ABAQUE}

Outre sa rapidité d'emploi et ses limites symétriques, l'abaque fournit immédiatement la précision de la mesure du peuplement.

ROBSON et REGIER (1964) avaient montré l'intérêt de connaitre le niveau de précision de la mesure du peuplement en vue de mieux conduire son étude. suivants :

Ils recommandaient d'atteindre ou de se contenter d'atteindre les niveaux

- 10 pour cent pour les études scientifiques

- 25 pour cent pour les aménagements

- 50 pour cent pour les prospections.

et proposaient une méthode pour déterminer le nombre de poissons à marquer afin d'obtenir le niveau de précision choisi à partir d'abaques utilisant $\mathrm{P}$ et $\mathrm{m}$.

Grâce au nouvel abaque, le niveau de précision résultant des deux premières pêches peut être déterminé sur le terrain. S'il est inférieur à celui qui serait nécessaire à l'objectif poursuivi, on peut avant de les remettre à l'eau marquer les poissons de la seconde pêche et procéder à une troisième pêche.

Nous n'avons considéré dans le present travail que le cas de la méthode de PETERSEN appliquée aux poissons, mais il va sans dire que l'abaque présenté peut être utilisé pour les autres espèces animales. 


\section{BIBLIOGRAPHIE}

CHAPMAN D.C., 1951. Some properties of the hypergeometrical distribution with applications to zoological censuses. Univ. Calif. Publs. Statist., 1 (7), 131-160.

CLOPPER C.J., PEARSON E.S., 1934. The use of confidence or fiducial limits illustrated in the case of the binomial. Biometrika, 26, 404-413.

CUINAT R., DUMAS J., TIMMERMANS J., ARRIGNON J., TUFFERY G., 1975 a. Diagnose écologique en cours d'eau à salmonides. Méthode et exemple. EIFAC Tech. Pap., 22, 122 p.

CUINAT R., DUMAS J., TIMMERMANS J., ARRIGNON J., TUFFERY G., 1975 b. Ecological diagnosis in Salmonid streams. Method and example. EIFAC Tech. Pap., 22, 122 p.

ROBSON D.S., REGIER H.A., 1964. Sample size in PETERSEN mark-and-recapture experiments. Trans. Am. Fish. Soc., 93 (3), 215-226.

TIMMERMANS J.A., 1957. Estimation des populations piscicoles. Applications aux eaux courantes rhéophiles. Trav. Stn Rech. Groenendaal, D, 21, $84 \mathrm{p}$.

VINCENT R., 1971. River electrofishing and fish populations estimates. Progve Fish. Cult., 33 (3), 163-169. 